\title{
Including Cognitive Aspects in Multiple Criteria Decision Analysis
}

\author{
João Carneiro · Luís Conceição · Diogo Martinho · \\ Goreti Marreiros · Paulo Novais
}

Received: date / Accepted: date

\begin{abstract}
Many Multiple Criteria Decision Analysis (MCDA) methods have been proposed over the last decades. Some of the most known methods share some similarities in the way they are used and configured. However, we live in a time of change and nowadays the decision-making process (especially when done in group) is even more demanding and dynamic. In this work, we propose a Multiple Criteria Decision Analysis method that includes cognitive aspects (Cognitive Analytic Process). By taking advantage of aspects such as expertise level, credibility and behaviour style of the decision-makers, we propose a method that relates these aspects with problem configurations (alternatives and criteria preferences) done by each decision-maker. In this work, we evaluated the Cognitive Analytic Process (CAP) in terms of configuration costs and the capability to enhance the quality of the decision. We have used the satisfaction level as a metric to compare our method with other known MCDA methods in literature (Utility function, AHP and TOPSIS). Our method proved to be capable to achieve higher satisfaction levels compared to other MCDA methods, especially when the decision suggested by CAP is different from the one proposed by those methods.
\end{abstract}

Keywords Multiple Criteria Decision Analysis · Cognitive decision-making · Group decision-making $\cdot$ Behavioural decision theory $\cdot$ AHP $\cdot$ TOPSIS

João Carneiro $(\varangle) \cdot$ Luís Conceição · Diogo Martinho · Goreti Marreiros

GECAD - Research Group on Intelligent Engineering and Computing for Advanced Innovation and Development, Institute of Engineering, Polytechnic of Porto, Porto 4200-072, Portugal

E-mail: jomrc@isep.ipp.pt

Luís Conceição

E-mail: 1071223@isep.ipp.pt

Diogo Martinho

E-mail: diepm@isep.ipp.pt

Goreti Marreiros

E-mail: mgt@isep.ipp.pt

João Carneiro · Paulo Novais

ALGORITMI Centre, University of Minho, Guimarães 4800-058, Portugal

E-mail: pjon@di.uminho.pt 


\section{Introduction}

Making decisions is intrinsic to the human being. Each action, more or less significant, conscious or unconsciously, results in a decision. Therefore, we could say we are all natural decision-makers (Saaty, 2008). The decision-making process can be seen as selecting one or more alternatives to be the solution for a certain problem/task (Chen, 2000).

Nowadays most of the decision-making processes happening in organizations are done in group (Luthans, 2011; Simon, 1965). There are many advantages related to group decisionmaking: to improve the quality of the decision, to share workloads, to gain support among stakeholders, to train less experienced group members and due to the majority of organograms existing nowadays (Dennis, 1996; Huber, 1984). However, due to market globalization and the firms' internationalization it is very hard to match each top managers' agenda, making it difficult for them to meet in the same space or time (Carneiro et al, 2015d). Therefore, it is no surprise that more techniques, models, methods and tools that support groups and individuals in the decision-making process are being developed.

Multiple Criteria Decision Analysis (MCDA) methods are one of the techniques used to support the decision-making process. MCDA is considered appropriate to deal with conflicting opinions and also qualitative and quantitative objectives (Ram et al, 2011; Golmohammadi and Mellat-Parast, 2012). MCDA provides a framework which deals with complex decision problems. In order to reach a consensus, a MCDA framework allows decision-makers to share information through problem configurations (Dehe and Bamford, 2015). Throughout the last decades, a wide variety of analytical models have been proposed in literature (Tavana et al, 2010). Some of the most acknowledged MCDA methods in literature are: ER, ELECTRE, PROMETHEE, AHP, ANP, TOPSIS, MACBETH (Figueira et al, 2005). Even though there is a very decent amount of work proposed under the topic of MCDA, the issues associated to MCDA are easy to identify. Tavana, Sodenkamp, and Suhl (2010) have identified certain issues such as: deficiencies when considering objective and subjective criteria, imprecise information due to the lack of the decision-makers' expertise, unavailability of data and time constraint, etc. Besides these issues, we can still include the lack of information about each decision-maker (Levy, 2007), the difficulty or impossibility for each decision-maker to express how he intends to face the decision-making process (Rahim and Magner, 1995) and the difficulty (the cost) associated to problems configuration (Dehe and Bamford, 2015).

The area of decision-making has always been very studied in literature. Since the early days of psychology and economy there has been a clear interest to study the topic of decision-making (Edwards, 1954; Simon, 1959, 1986). If economists have always looked at decision-making in a completely rational perspective, psychologists look at decisionmaking in a perspective which allows them to understand the individual judgement and the decision-making concerning both irrational and rational aspects of behaviour (Simon, 1986). It is evident that most of the existent MCDA methods have been based in an economic perspective which we could call as the rational choice. In 1979, Herbert Simon (Simon, 1979) pointed the interest of economists to explore domains that belonged, traditionally, to political sciences, sociology, and psychology. This interest is very similar to what happens nowadays between computer science researchers and those same domains (Castelfranchi, 1994; Smith and Conrey, 2007; Falcone and Castelfranchi, 2001; Bates et al, 1994; Ogiela and Ogiela, 2014b,a). It is known that cognitive aspects are present and affect the individual during the decision-making process (Schwarz, 2000). Therefore it is assumed to be necessary to consider cognitive aspects in order to make a correct representation of the interests of decision-makers. With that being said, it can then claim that including cognitive aspects 
allow obtaining better decisions which are fundamentally more adjusted to the real interests of decision-makers.

In this work, we present a new MCDA method named as Cognitive Analytical Process (CAP). CAP allowed decision-makers to represent their preferences about a problem (criteria and alternatives) and also represent what we referred as their intentions, being directed towards supporting group decision-making. Decision-makers could: express their intentions by selecting a behaviour style that better represents how they intend to face the decisionmaking process, select an expertise level about the topic being discussed and select which decision-makers involved in the same process they consider to be more credible. CAP is an analytic method that aims to provide a decision based on the values mentioned in the previous configurations and that maximizes the satisfaction level of the group and of each one of the decision-makers.

The rest of the paper is organized as follows: in the next section is presented the background, where we enumerated the essential methods to define our proposal, in the following section (3) we presented our approach, where the Cognitive Analytic Process is described and the model is formulated. In Section 4 it is shown the evaluation and results of this work and in Section 5 the discussion is presented. Finally, some conclusions were taken in Section 6 , along with the work to be done hereafter.

\section{Background}

It is easy to find in literature several authors which refer to the importance of psychological and cognitive aspects in the decision-making context (Schwenk, 1988; Frith and Singer, 2008; Schwarz, 2000). Moreover, there are authors which defend that the quality of the decision decreases when theses aspects are not considered (Simon, 1987a). Some authors consider that including cognitive and psychological aspects help to achieve decisions with higher quality (Simon, 1987a; Fenton-O'Creevy et al, 2011). Having all these evidences into account it becomes clear how a decision made using automatic mechanisms can be affected. There are many factors and knowledge which may be lost when the human interaction stops existing, even if there can be other advantages associated (Kiesler et al, 1984). By using a typical multi-criteria problem configuration, how will the system manager knowledge and expertise levels of each decision-maker? Will it be fair to weight all configurations as the same? How to deal with different intentions which the decision-maker may have in each decision-making scenario? In this work we intend to answer these questions as well as verify if our method manages to achieve higher results (compared to other methods) by considering some of these points.

In this section, the models used by CAP are presented and allow us to better understand how it works. Besides this, it is explained the reasons why behaviour styles, credibility and expertise are considered by CAP as well as how they relate with the topic of group decisionmaking. All these points have been discussed in some of our most recent works (Carneiro et al, 2015c,b,a; Martinho et al, 2015), and they have been used because according to the work published in (Carneiro et al, 2015c) they are part of a multi-criteria configuration template that proved to be very easy and fast to configure. This fact was important due to big concerns related to the usability and the resources needed to use CAP. The description next presented for each point is based in the analysis done previously in (Carneiro et al, 2015c). 


\subsection{Behaviour Style}

The behaviour style can be seen as the expected behavior or the desirable behaviour. In some of our previous works, we studied the difference between using the decision-maker's personality or to create the possibility for the decision-maker to select the conflict style or the behaviour that he intended to use (to read more about this see Martinho, Carneiro, Marreiros, and Novais (2015)). During our life, we are constantly presented with the need to make decisions. Some decisions are less relevant, others are not. Fundamentally, when we are taking decisions in group, and independently of our personality, we express and behave differently according to different situations such as: the topic of the problem to be solved, to consider the others decision-makers as more expert than us, to intend to be pleasant and try to pursue other decision-makers' goals, the level of the interest on the topic, the mood, etc. This means that in a hypothetical scenario where a decision-maker and his family are deciding from a group of alternatives to choose a restaurant to celebrate his son's birthday, maybe his main concern is to satisfy his son's preferred alternative. However, he still has his own preferences regardless of the kind of behaviour he shows during the negotiation process. In (Martinho et al, 2015), 5 behaviour styles were considered: Dominating, Integrating, Compromising, Obliging and Avoiding. These styles differentiate from each other by what was thought to be 4 essential dimensions for this context:

1. Concern for Self - This dimension was related to the individual's concern for his own opinion above the others since he was likely to adapt a more one-sided attitude during the decision-making process by making statements, questions and requests that detailed that opinion;

2. Concern for others - This dimension was related to the individual's concern for other individuals' opinion. He adapted a more altruist attitude during the decision-making process, tried to understand other opinions and made an effort to reach a decision that benefited or pleased most of the participants;

3. Activity - This dimension was related to the effort put into the decision-making process by the individual, meaning that the more active an individual was, the more questions and statements and requests he was likely to make;

4. Resistance to change - This dimension was related to how hard or easy it was for an individual to accept other opinions.

In Table 1, we describe each behaviour style by providing a value for every dimension (Concern for Self, Concern for Others, Activity and Resistance to Change) mentioned before. The numeric values (1, 2 or 3 for low, mid and high respectively) given to each dimension are correlated with the definitions established by Rahim and Magner (1995) (to learn more about this correlation see Rahim and Magner (1995); Martinho, Carneiro, Marreiros, and Novais (2015)).

\subsection{Credibility}

There is not a universally accepted definition for credibility. Besides that, the study of credibility is highly multi-disciplinary and some of the suggested definitions are related to their area of operation (Flanagin and Metzger, 2008). In our work, it was considered the definition proposed by Flanagin and Metzger (2008) when they said that "the overarching view is that credibility is the believability of a source or message, which is made up of two primary dimensions: trustworthiness and expertise". Trustworthiness was related with subjective components while expertise was related with more objective components. The notion of 
Table 1: Behaviour styles and corresponding dimensions, adapted from Martinho, Carneiro, Marreiros, and Novais (2015)

\begin{tabular}{lllll}
\hline Behaviour Style & Concern for Self & Concern for Others & Activity & Resistance to Change \\
\hline Dominating & 3 & 1 & 3 & 3 \\
Integrating & 3 & 3 & 3 & 3 \\
Compromising & 2 & 2 & 2 & 2 \\
Obliging & 1 & 3 & 1 & 1 \\
Avoiding & 1 & 1 & 1 & 1 \\
\hline
\end{tabular}

credibility was related with many other concepts including trust, reliability, accuracy, quality, authority, reputation, competence, etc (Flanagin and Metzger, 2008). In our work, the main idea was to let a decision-maker choose which other decision-maker he considered to be credible towards a certain topic. This credibility evaluation was related with the concepts mentioned above and would be the reason why a decision-maker might consider another decision-maker to be credible for a topic and not for a different topic (for example, with the related expertise level recognized for that decision-maker), and also why a decision-maker might consider another decision-maker always credible despite of the topic's difference (for example, due to reasons such as authority, reputation, etc.).

\subsection{Expertise}

Expertise is considered as one of the credibility dimensions (affected by objective components) (Flanagin and Metzger, 2008). In our work, it was intended to allow the decisionmaker to make a self-evaluation about his expertise level for the topic at hand. It was considered the existence of five different expertise levels: Expert, High, Medium, Low and Null (the definition of the expertise levels definitions and values can be consulted in Table 2). Why is this information relevant and how can it be used? This kind of information could have many applications. One could be to use it to compare the self-evaluation made by the decision-maker for its expertise level with its credibility which is recognized by other decision-makers, allowing further conclusions to be made. This information could also be used to compare the self-evaluation with the chosen conflict style.

Table 2: Expertise Levels

\begin{tabular}{lll}
\hline Expertise Level & Inverse of Expertise Level & Definition \\
\hline 5 & 1 & Expert \\
4 & 2 & High \\
3 & 3 & Medium \\
2 & 4 & Low \\
1 & 5 & Null \\
\hline
\end{tabular}




\section{Cognitive Analytic Process}

In this section, we present all the steps necessary to formalize the Cognitive Analytic Process. This formalization is based in the general concept of multi-criteria decision-making and was first inspired in the works of other authors (Bozóki et al, 2013; Wang, 2012; Ahn and Choi, 2012; Kou and Wu, 2014). For each step, we present definitions related with multicriteria decision-making and our model. In Table 3 it is presented all notations necessary to better understand our formalization.

Table 3: Notation correspondence

\begin{tabular}{ll}
\hline Terminology & Notation \\
\hline Decision matrix & $D$ \\
Set of criteria & $C$ \\
Set of alternatives & $A$ \\
Criterion & $C_{i}$ \\
Alternative & $A_{i}$ \\
Decision-maker & $D m_{i}$ \\
Weight & $W$ \\
Criterion importance & $I m p c_{i}$ \\
Set of decision-makers & $D M$ \\
Preferences of a set of decision-makers & $W D M$ \\
Criteria preference matrix & $C P$ \\
Set of sums of the best criteria weighting & $H S c$ \\
\hline
\end{tabular}

\section{Step 1 Multi-Criteria Problem Definition}

The first step of CAP was to define the multi-criteria problem. Therefore:

Definition 1 Let $D$ be a decision matrix, where $D=A \times C=\left[\begin{array}{cccc}c_{1_{a_{1}}} & c_{2_{a_{1}}} & \ldots & c_{n_{a_{1}}} \\ c_{1_{a_{2}}} & c_{2_{a_{2}}} & \ldots & c_{n_{a_{2}}} \\ \vdots & \vdots & \ldots & \vdots \\ c_{a_{m}} & c_{a_{m}} & \ldots & c_{n_{a_{m}}}\end{array}\right]$ and consists of:

- a set of criteria $C=\left\{c_{1}, c_{2}, \ldots, c_{n}\right\}, n>0$;

- a set of alternatives $A=\left\{a_{1}, a_{2}, \ldots, a_{m}\right\}, m>0$;

Rule $1 \forall a_{i} \in A, \forall c_{j} \in C, c_{j a_{i}} \in D$

Each alternative $a_{j} \in A$ is related with each criteria $c_{i} \in C$. There cannot be an existing alternative with values for criteria that are not considered in the problem.

Definition 2 A criterion $c_{i}=\left\{i d_{c_{i}}, v_{c_{i}}, m_{c_{i}}\right\}$ consists of:

- $\forall c_{i} \in C, i \in\{1,2, \ldots, n\}$

- $i d_{c_{i}}$ is the identification of a particular criterion;

$-v_{c_{i}}$ is the value of a particular criterion (Numeric, Boolean or Classificatory);

- $m_{c_{i}}$ is the greatness associated with the criterion (Maximization, Minimization, Positivity, Negativity and without value). 
Example 1 Let us consider criteria Price, Size and Quantity. This criteria can be defined as follows:

- $c_{1}=\{$ Price,Numeric,Minimization $\}$;

- $c_{2}=\{$ Size,Numeric,Maximization $\}$

$-c_{3}=\{$ Quantity, Numeric,Maximization $\}$.

Definition 3 An alternative $a_{i}=\left\{i d_{a_{i}},\left[c_{a_{a_{i}}}, c_{2_{a_{i}}}, \ldots, c_{n_{a_{i}}}\right]\right\}$ consists of:

- $\forall a_{i} \in A, i \in\{1,2, \ldots, m\}$;

- $i d_{a_{i}}$ is the identification of a particular alternative;

- $\left[c_{1_{a_{i}}}, c_{2_{a_{i}}}, \ldots, c_{n_{a_{i}}}\right]$ is the instantiation of each criterion.

Example 2 For the same example, let us consider three alternatives. Each alternative is defined as follows:

- $a_{1}=\{$ alternative $1,[10000 \$, 100 \mathrm{~cm}, 90]\}$

$-a_{2}=\{$ alternative $2,[12500 \$, 150 \mathrm{~cm}, 50]\}$

$-a_{3}=\{$ alternative $3,[15000 \$, 170 \mathrm{~cm}, 70]\}$.

\section{Step 2 Criteria Weighting}

The second step of CAP was to define the weights given by each decision-maker towards each criterion and alternative.

Definition 4 Let $w_{d m_{i_{c}}}$ be the weight or preference given to the criterion $c_{j}$ by a decisionmaker $d m_{i}$ and $c_{n} \in C$.

Example 3 For the same example a possible weight given to each criterion by a decisionmaker $d m_{i}$ could be $W_{d m_{1_{c_{1}}}}=0,4, W_{d m_{1_{c_{2}}}}=0,6, W_{d m_{1_{c_{3}}}}=0,8$.

Rule 2 A decision-maker $d m_{i}$ may define a set of $W_{d m_{i}}$ weights where:

- $W_{d m_{i}}=\left\{w_{d m_{i_{1}}}, w_{d m_{i_{2}}}, \ldots, w_{d m_{i_{n}}}\right\}, n>0, \forall j \in\{1,2, \ldots, n\}, 0 \leq w_{d m_{i_{c_{n}}}} \leq 1 ;$

$-\left\langle W_{d m_{i}}\right\rangle=\langle C\rangle$.

We defined a function which returned the difference between the maximum and minimum weights that belonged to a set of $W_{d m_{i}}$ weights.

$$
F_{D i f}: W_{d m_{i}}\left\{\begin{array}{l}
\max \left(W_{d m_{i}}\right)-\min \left(W_{d m_{i}}\right), \quad \text { if } \max \left(W_{d m_{i}}\right) \neq \min \left(W_{d m_{i}}\right) \\
\max \left(W_{d m_{i}}\right)
\end{array}\right.
$$


This difference was classified in 5 different levels in the following order according to Table 4. The values presented in the table for each level are related with the fact that we are dealing with 5 criteria classifications (VIC, IC, MD, NIC and INC). Since each criteria weighting is done in a scale of $[0,1]$ the minimum difference between two criteria is less than 0,2 and the maximum difference is greater than 0,8 . Measuring the difference (using function $F_{D i f}$ ) between the criterion with more weight and the criterion with less weight we can obtain (according to Table 4) the " $l$ " value. This value is then used in the algorithm to measure the classification done for each criterion (imp).

Table 4: $F_{D i f}$ levels

\begin{tabular}{ll}
\hline Level $(l)$ & $F_{\text {Dif }}$ \\
\hline 5 & $\geq 0,80$ \\
4 & $\geq 0,60$ \\
3 & $\geq 0,40$ \\
2 & $\geq 0,20$ \\
1 & $<0,20$ \\
\hline
\end{tabular}

Example 4 For the same example we know that $W_{d m_{1}}=\{0,4 ; 0,6 ; 0,8\}$.

We classified the importance $i m p_{c_{j_{d m_{i}}}}$ for each criterion $c_{j} \in C$ to the decision-maker $d m_{i}$ by using the following algorithm 1 .

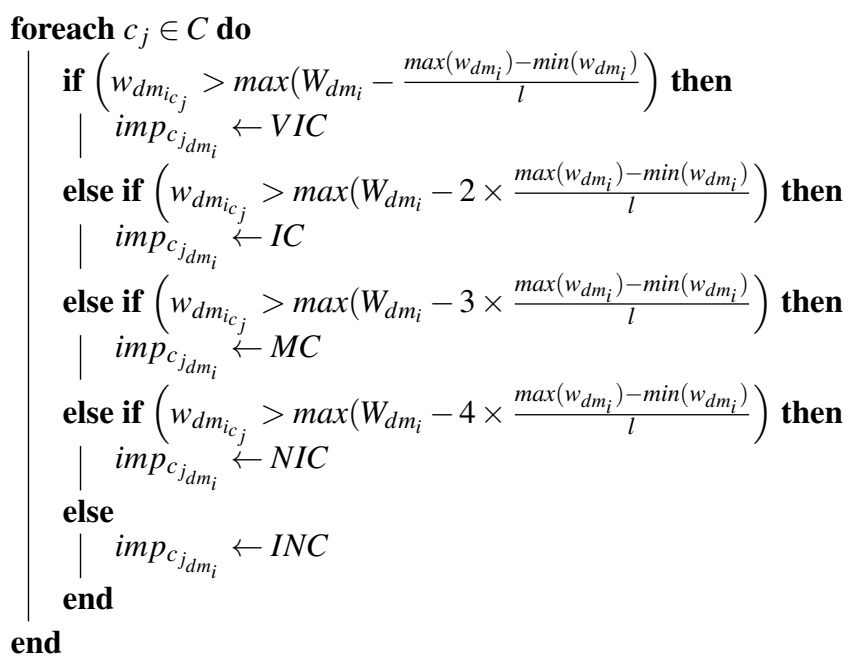

Algorithm 1: Importance classification algorithm

The importance given to a criterion depended on $l$ and varied in the following order, represented in Table 5:

Example 5 For the same example, let us consider the decision-maker $d m_{1}$, the set of criteria $C=\left\{c_{1}, c_{2}, c_{3}\right\}$, and the set of weights $W_{d m_{1}}=\{0,4 ; 0,6 ; 0,8\} . F_{D i f}=0,80-0,40=0,40$. Looking at Table 5 we now know that $l=3$. We can use algorithm 1 to classify each criterion as: 
Table 5: Criterion importance

\begin{tabular}{ll}
\hline${ }^{i m p_{{ }_{j_{d m_{i}}}}}$ & Definition \\
\hline VIC & Very Important Criterion \\
IC & Important Criterion \\
MC & Medium Criterion \\
NIC & Not Important Criterion \\
INC & Insignificant Criterion \\
\hline
\end{tabular}

$$
\begin{gathered}
w_{d m_{1_{c_{1}}}}>0,8-4 \times \frac{0,8-0,4}{3} \equiv 0,4>0,266 \rightarrow i m p_{c_{1_{d m_{1}}}}=N I C ; \\
w_{d m_{1_{c_{2}}}}>0,8-2 \times \frac{0,8-0,4}{3} \equiv 0,6>0,533 \rightarrow i m p_{c_{2_{d m_{1}}}}=V I C ; \\
w_{d m_{1_{c_{3}}}}>0,8-\frac{0,8-0,4}{3} \equiv 0,8>0,666 \rightarrow i m p_{c_{3_{d m_{1}}}}=V I C .
\end{gathered}
$$

Definition 5 Let $W_{D M}$ be the preferences of a set of decision-makers $D M$ where:

$$
W_{D M}=\left\{W_{d m_{1}}, W_{d m_{2}}, \ldots, W_{d m_{z}}\right\}, z>0 ;
$$

Definition 6 Let $D M$ be a set of decision-makers where $D M=\left\{d m_{1}, d m_{2}, \ldots, d m_{k}\right\}, k>0$.

Definition 7 Let $C P$ be a criteria preference matrix, where:

$$
C P=C \times W_{D M}=\left[\begin{array}{cccc}
w_{1_{1}} & w_{2_{c_{1}}} & \ldots & w_{z_{c_{1}}} \\
w_{1_{c_{2}}} & w_{2_{c_{2}}} & \ldots & w_{z_{c_{1}}} \\
\vdots & \vdots & \ldots & \vdots \\
w_{1_{c_{m}}} & w_{2_{c_{m}}} & \ldots & w_{z_{c_{m}}}
\end{array}\right]
$$

Example 6 For the same example let us now consider two more decision-makers $d m_{2}$ and $d m_{3}, D M=\left\{d m_{1}, d m_{2}, d m_{3}\right\}$, two more sets of weights $W_{d m_{2}}=\{0,2 ; 0,8 ; 0,45\}, W_{d m_{3}}=$ $\{0,7 ; 0,55 ; 0,6\}$.

We combined all three sets of weights into $W_{D M}=\left\{W_{d m_{1}}, W_{d m_{2}}, W_{d m_{3}}\right\}$ and obtained the following preference matrix:

$$
C P_{D M}=C \times W_{D M}=\left[\begin{array}{lll}
W_{d m_{1 c_{1}}} & W_{d m_{2 c_{1}}} & W_{d m_{3 c_{1}}} \\
W_{d m_{1 c_{2}}} & W_{d m_{2_{c_{2}}}} & W_{d m_{3 c_{2}}} \\
W_{d m_{1_{c_{3}}}} & W_{d m_{2_{c_{3}}}} & W_{d m_{3 c_{3}}}
\end{array}\right]=\left[\begin{array}{lll}
0,40 & 0,20 & 0,70 \\
0,60 & 0,80 & 0,55 \\
0,80 & 0,45 & 0,60
\end{array}\right]
$$

We applied Algorithm 1 to measure the importance given to each criterion by each decision-maker and obtained:

$$
C P_{D M}=\left[\begin{array}{lll}
0,40 & 0,20 & 0,70 \\
0,60 & 0,80 & 0,55 \\
0,80 & 0,45 & 0,60
\end{array}\right]=\left[\begin{array}{lll}
M C & I N C & V I C \\
V I C & V I C & I C \\
V I C & M C & V I C
\end{array}\right]
$$

We defined a function which returns 0 or 1 depending if a $w_{z_{c}}$ was either VIC, IC, or not.

$$
F_{\text {highimp }}: W_{z_{c_{m}}}\left\{\begin{array}{l}
1, \\
0
\end{array} \text { if } w_{z_{c_{m}}}=V I C \vee w_{z_{c_{m}}}=I C\right.
$$


Definition 8 Let $H S_{c}$ be a set of sums of the best criteria weighting $H S_{c}=\left\{h s_{c_{1}}, h s_{c_{2}}, h s_{c_{3}}, \ldots, h s_{c_{m}}\right\}$, where:

$$
\forall i \in\{1,2, \ldots, m\}, h s_{c_{i}}=\sum_{w_{z_{i}} \in C P} F_{\text {highimp }}\left(w_{z_{c_{i}}}\right)
$$

is the sum of all elements in a criteria preference matrix $\mathrm{CP}$ which are either VIC or IC for each criterion $c_{m}$.

Example 7 For the same example using the criteria preference matrix $C P_{D M}$ we would obtain the following sums:

$$
\begin{gathered}
h s_{c_{1}}=\sum_{w_{z_{c_{1}}} \in C P} F_{\text {highimp }}\left(w_{z_{c_{1}}}\right)=0+0+1=1 \\
h s_{c_{2}}=\sum_{w_{z_{c_{2}}} \in C P} F_{\text {highimp }}\left(w_{z_{c_{2}}}\right)=1+1+1=3 \\
h s_{c_{3}}=\sum_{w_{z_{3}} \in C P} F_{\text {highimp }}\left(w_{z_{c_{3}}}\right)=1+0+1=2 \\
H S_{c}=\{1,3,2\}
\end{gathered}
$$

\section{Step 3 Credibility and Expertise Readjustment}

The third and final step of CAP was to measure the added value to criteria preferences depending on the credibility and expertise of the decision-makers. For this, we used a criteria preference matrix $C P_{D M C r e d b l e}$ which contained criteria weights given by each credible decision-maker. Depending on the conflict style (see Table 1) of the decision-maker $d m_{i}$ the following formula was used to readjust the preference for criterion $c_{j}$ :

$$
\forall d m_{i} \in D M, \forall c_{j} \in C, c r w_{d m_{i_{j}}}=\frac{F_{h i g h i m p}\left(W_{d m_{i_{c_{j}}}}\right) \times C S_{d m_{i}}+\left(\frac{T P}{N D}\right) \times C O_{d m_{i}}}{C S_{d m_{i}}+C O_{d m_{i}}}
$$

Where:

- F highimp $\left(W_{d m_{i_{c_{j}}}}\right)$ returns 1 or 0 depending on the importance given to criterion $c_{j}$ by decision-maker $d m_{i}$;

- $C S_{d m_{i}}$ is the value of Concern for Self $[1,2,3]$ of the decision-maker $d m_{i}$;

- TP is the total sum of the weights given to criterion $c_{j}$ by each credible decision-maker;

- ND is the total number of credible decision-makers;

- $C O_{d m_{i}}$ is the value of Concern for Others $[1,2,3]$ of the decision-maker $d m_{i}$.

Example 8 For the same example let us consider that decision-maker $d m_{1}$ thinks $d m_{2}$ and $d m_{3}$ are credible, DMCredible Dm $_{1}=\left\{d m_{2}, d m_{3}\right\}$. Let us also consider that $d m_{1}$ has the conflict style 'Obliging'. $C P_{D_{M M} \text { Cedible }_{d m_{1}}}$ is:

$$
C P_{\text {DMCredible }_{d m_{1}}}=\left[\begin{array}{ll}
0,20 & 0,70 \\
0,80 & 0,55 \\
0,45 & 0,60
\end{array}\right]=\left[\begin{array}{ll}
I N C & V I C \\
V I C & I C \\
M C & V I C
\end{array}\right]
$$


We used formula 1 to readjust the weight given to each criterion by decision-maker $d m_{1}$ as:

$$
\begin{gathered}
c r w_{d m_{1_{c_{1}}}}=\frac{0 \times 1+\left(\frac{0,20+0,70}{2}\right) \times 3}{1+3}=\frac{1,35}{4}=0,3375 \\
\operatorname{crw}_{d m_{1_{c_{2}}}}=\frac{1 \times 1+\left(\frac{0,80+0,55}{2}\right) \times 3}{1+3}=\frac{3,025}{4}=0,75625 \\
c r w_{d m_{1_{c_{3}}}}=\frac{1 \times 1+\left(\frac{0,45+0,60}{2}\right) \times 3}{1+3}=\frac{2,575}{4}=0,64375
\end{gathered}
$$

Next, we redjusted the value of $c r w_{d m_{i_{j}}}$ with the level of expertise of the decision-maker $d m_{i}$ and the inverse of the expertise level (see Table 2) using the following formula:

$$
\forall d m_{i} \in D M, \forall c_{j} \in C, n e w w_{d m_{i_{j}}}=\frac{c r w_{d m_{i_{j}}} \times e_{d m_{i}}+\left(\frac{T P}{N D}\right) \times e_{d m_{i}}^{\prime}}{e_{d m_{i}}+e_{d m_{i}}^{\prime}}
$$

Where:

- $c r w_{d m_{i c_{j}}}$ is the readjusted weight given to criterion $c_{j}$ by decision-maker $d m_{i}$ using formula 1;

- $e_{d m_{i}}$ is the expertise level of decision-maker $d m_{i}$;

- TP is the total sum of the weights given to criterion $c_{n}$ by each credible decision-maker;

- ND is the total number of credible decision-makers;

$-e_{d m_{i}}^{\prime}$ is the inverse of the expertise level of decision-maker $d m_{i}$.

Example 9 For the same example, let us consider that decision-maker $d m_{1}$ has an expertise level $e_{d m_{1}}=4$. Using formula 2 we will obtain:

$$
\begin{gathered}
n e w w_{d m_{1_{c_{1}}}}=\frac{0,3375 \times 4+\left(\frac{0,20+0,70}{2}\right) \times 2}{4+2}=\frac{2,25}{6}=0,375 \\
n e w w_{d m_{1_{c_{2}}}}=\frac{0,75625 \times 4+\left(\frac{0,80+0,55}{2}\right) \times 2}{4+2}=\frac{4,375}{6}=0,7292 \\
n e w w_{d m_{1_{c_{3}}}}=\frac{0,64375 \times 4+\left(\frac{0,45+0,60}{2}\right) \times 2}{4+2}=\frac{3,625}{6}=0,6041
\end{gathered}
$$

We measured the final classification given by a decision-maker $d m_{i}$ with a criteria preference matrix $C P_{D M C r e d i b l e} d_{i}$ for each criterion $c_{j}$ using the following formula:

$$
\forall d m_{i} \in D M, \forall c_{j} \in C, \text { final }_{d m_{i_{j}}}=\left(\frac{h s_{c_{j}}}{N D}\right) \times\left(\frac{T P}{N D}\right)+\left(1-\frac{h s_{c_{j}}}{N D}\right) \times n e w w_{d m_{i_{j}}}
$$

Where:

- $n e w w_{d m_{i, c_{j}}}$ is the readjusted weight given to criterion $c_{j}$ by decision-maker $d m_{i}$ using formula 2;

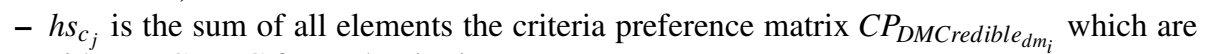
either VIC or IC for each criterion $c_{j}$;

- TP is total sum of the weights given to criterion $c_{j}$ by each credible decision-maker; 
- $N D$ is the total number of credible decision-makers.

Example 10 For the same example decision-maker $d m_{1}$ reclassifies each criterion as:

$$
\begin{gathered}
\text { final }_{d m_{1_{c_{1}}}}=\left(\frac{1}{2}\right) \times\left(\frac{0,20+0,70}{2}\right)+\left(1-\frac{1}{2}\right) \times 0,375=\frac{0,90}{4}+\frac{0,375}{2}=0,4125 \\
\text { final }_{d m_{1_{c_{2}}}}=\left(\frac{2}{2}\right) \times\left(\frac{0,80+0,55}{2}\right)+\left(1-\frac{2}{2}\right) \times 0,7292=\frac{1,35}{2}=0,675 \\
\text { final }_{d m_{1_{c_{3}}}}=\left(\frac{1}{2}\right) \times\left(\frac{0,45+0,60}{2}\right)+\left(1-\frac{1}{2}\right) \times 0,6041=\frac{1,05}{4}+\frac{0,6041}{2}=0,56455
\end{gathered}
$$

Looking only at $W_{d m_{1}}$ initial setup we have $W_{d m_{1}}=\{0,4 ; 0,6 ; 0,8\}$, and therefore $w_{d m_{1_{3}}}>$ $w_{d m_{1_{c_{2}}}}>w_{d m_{1_{c_{1}}}}$. Using CAP method which takes into account the preferences, the conflict style and the expertise level of the decision-maker as well as the credibility of other decisionmakers, the predicted classification will be final $w_{d m_{1_{c_{2}}}}>$ final $w_{d m_{1_{c_{3}}}}>$ finalw $w_{d m_{1_{1}}}$.

\section{Experiments}

To test the Cognitive Analytic Process (CAP), we developed a prototype which could load a multi-criteria problem, and analyzed and compared each solution found with all chosen and implemented methods including the Analytic Hierarchy Process (AHP) (Saaty, 1988), TOPSIS (Tzeng and Huang, 2011) and a typical utility function method (UF).

We opted to compare CAP with these three methods since our approach followed a very different perspective from what is currently observed under the topic of MCDA. Therefore we thought it was relevant to compare CAP with some of the more recognized methods that gave origin to many existing proposals we know today. Since our main focus was to support group decision-making, the developed prototype was also a multi-agent system where each agent represented a real decision-maker.

The adopted multi-criteria problem was to choose a medication. All considered criteria and alternatives were selected based on the work of De (1993). Each agent represented one member of the medical team. All alternatives were classified according to four criteria: efficacy, safety, convenience and low cost. These criteria were numeric and of maximization. In Table 6, all specifications are presented for each considered alternative (this information did not represent real data and was only used as an example for each performed simulation).

The satisfaction was used as a metric to evaluate the overall performance of the different methods. The satisfaction metric was used to understand the perception (by the decisionmaker) of the quality of the chosen alternative or the alternative supported by most agents at a certain time. For that, the notion of satisfaction that was used was the one proposed in (Carneiro et al, 2015a). It was the only satisfaction metric existing in literature adapted to this type of context. According to this model, the satisfaction of each decision-maker corresponded to the perception of the quality of the decision and was created based on assumptions which reflected what a decision-maker psychologically considered as a good decision. The satisfaction was measured in two parts (only CAP includes the second part). It was first measured objectively through the formulas 4, 5 and 6. 
Table 6: Multi-Criteria Problem

\begin{tabular}{lllll}
\hline & Efficacy & Safety & Convenience & Low Cost \\
\hline Antihistamines & 0,98 & 0,26 & 0,72 & 0,47 \\
Corticosteroids & 0,60 & 0,85 & 0,05 & 0,60 \\
$\beta$-adrenoceptor agonists & 0,38 & 0,51 & 0,64 & 0,42 \\
Methylxanthines & 0,91 & 0,07 & 0,15 & 0,82 \\
Anticholinergic & 0,21 & 0,89 & 0,76 & 0,26 \\
\hline
\end{tabular}

$$
\begin{gathered}
D_{\text {Lost }}=A l t_{F}-\text { Alt }_{P} \\
A_{\text {Conversion }}=2 \times A l t_{F}-1 \\
D_{\text {Satisfaction }}=1-\left|A_{\text {Conversion }}\right| \times D_{\text {Lost }}+A_{\text {Conversion }}
\end{gathered}
$$

Where:

- $D_{\text {Lost }}$ is the loss of decision maker's satisfaction in the difference between the assessments made for the alternative chosen by the group and for his preferred alternative. The loss is zero when the chosen alternative is the same as his preferred alternative;

- $A l t_{F}$ is the assessment made by the participant for the final alternative, alternative chosen by the group;

- $A l t_{P}$ is the assessment made by the participant for his preferred alternative;

- A Conversion is the conversion of the assessment made by the participant in the range $[-1,1]$.

The second part related the $D_{\text {Satisfaction }}$ and the behaviour defined by the decision-maker. In this second part, the satisfaction was measured according to the values of the agent's defined behaviour (agent's with defined behaviour follow the work proposed in Martinho, Carneiro, Marreiros, and Novais (2015)) for concern for self and concern for others dimensions. So, the $D_{\text {Satisfaction was remeasured using formula } 7 .}$

$$
D_{\text {Satisfaction }}=\frac{D_{\text {Satisfaction }} \times C S+O A A D_{\text {Satisfaction }} \times C O}{C S+C O}
$$

Where:

- $C S$ is the value of Concern for Self $[1,2,3]$;

- OAAD $D_{\text {Satisfaction }}$ is the average satisfaction of all remaining agents;

- $C O$ is the value of Concern for Others $[1,2,3]$.

In order to evaluate CAP, we created several simulation environments referring to the multi-criteria problem presented above. The first simulation environment tested 12 agents. For this environment 10000 simulations were run and in each simulation the 4 methods (CAP, TOPSIS, AHP and UF) were used to study and compare the results obtained using the same configuration parameters. Table 7 represents the main data retrieved in all those simulations. It was easy to observe that the data obtained using each method was very similar. We verified, however, agents' satisfaction throughout each simulation was higher when 
Table 7: Head table with the results obtained for each method throughout 10000 simulations.

\begin{tabular}{lrrrr}
\hline & CAP & TOPSIS & AHP & UF \\
\hline Average & 0,3309 & 0,3193 & 0,2966 & 0,3238 \\
Minimum & $-0,0858$ & $-0,0886$ & $-0,1776$ & $-0,1410$ \\
Maximum & 0,8119 & 0,7838 & 0,7838 & 0,7838 \\
Median & 0,3285 & 0,3183 & 0,2960 & 0,3203 \\
\hline
\end{tabular}

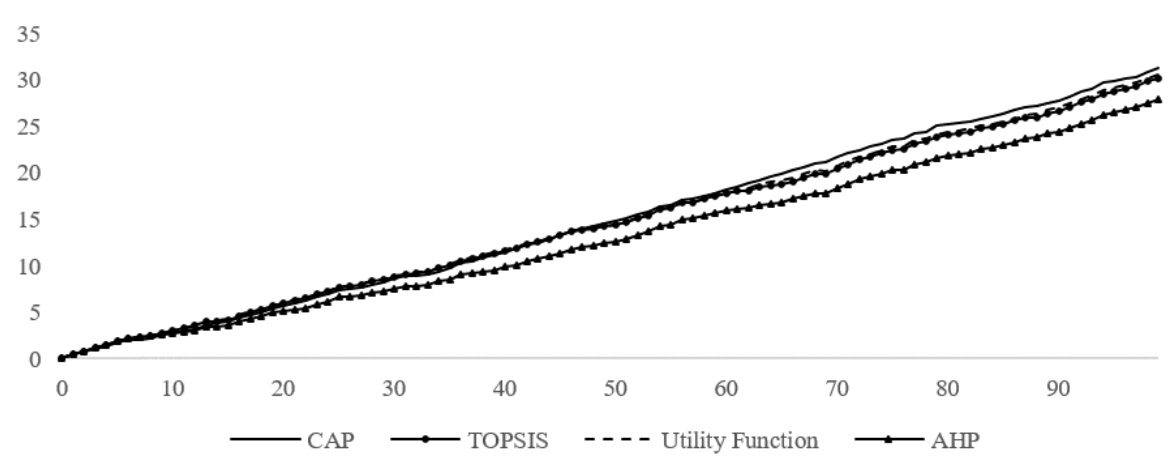

Fig. 1: Graphic with main comparisons taken with a sample of 100 simulations from 10000 total simulations

CAP was used. Besides this, CAP also included best results towards minimum, maximum and median values.

In order to know if the average satisfaction level obtained in CAP was indeed higher throughout each simulation, the added satisfaction levels are presented in Fig 1 (X-axis represents the simulation number and Y-axis represents the sum of the satisfaction level) for each method from a sample of 100 simulations. We verified that the values obtained in Table 7 corresponded to the growth presented by each method. As mentioned before the general data presented in each method was very similar. However, we intended to study the results for each method in situations where the chosen alternative or decision was different. More specific results were then presented which resulted from a direct comparison between CAP and all other considered methods.

As mentioned before the general data presented in each method was very similar. However, what we intended to study was the results for each method in situations where the chosen alternative or decision was different. The more specific results were then presented which resulted from a direct comparison between CAP and all other considered methods.

Table 8 shows the results collected for CAP and AHP. In 10000 simulations CAP obtained a higher satisfaction in 6241 occasions. The average, standard deviation, maximum, minimum and median values concern CAP when the satisfaction obtained was higher and corresponded to the difference of satisfaction between CAP and AHP (CAP - AHP). Likewise, when AHP obtained a higher satisfaction those values corresponded to the difference of satisfaction between AHP and CAP (AHP - CAP). It was verified that the average obtained in CAP was almost the double compared to the average obtained by AHP. The standard deviation value indicated that the average in AHP was less variable. The minimum, 
maximum and median values obtained by CAP were all higher compared to the values obtained by AHP.

Table 8: Comparison between CAP and AHP in 10000 simulations

\begin{tabular}{lrr}
\hline & CAP & AHP \\
\hline Count & 6241 & 3759 \\
Average & 0,0830 & 0,0464 \\
Standard Deviation & 0,0726 & 0,0375 \\
Maximum & 0,4408 & 0,2706 \\
Minimum & $2,3872 \times 10^{-6}$ & $4,5927 \times 10^{-6}$ \\
Median & 0,0614 & 0,0381 \\
\hline
\end{tabular}

CAP selected a different alternative from AHP in 3233 occasions. Fig 2a (X-axis represents the simulation number and Y-axis represents the satisfaction level) shows the results from a sample of 50 simulations. It was verified that the satisfaction obtained was almost always higher when CAP was used. The average satisfaction level obtained by agents in those 3233 simulations was of 0,2944, while AHP was only of 0,1995. Besides this, in 2609 of those 3233 simulations CAP obtained a higher satisfaction.
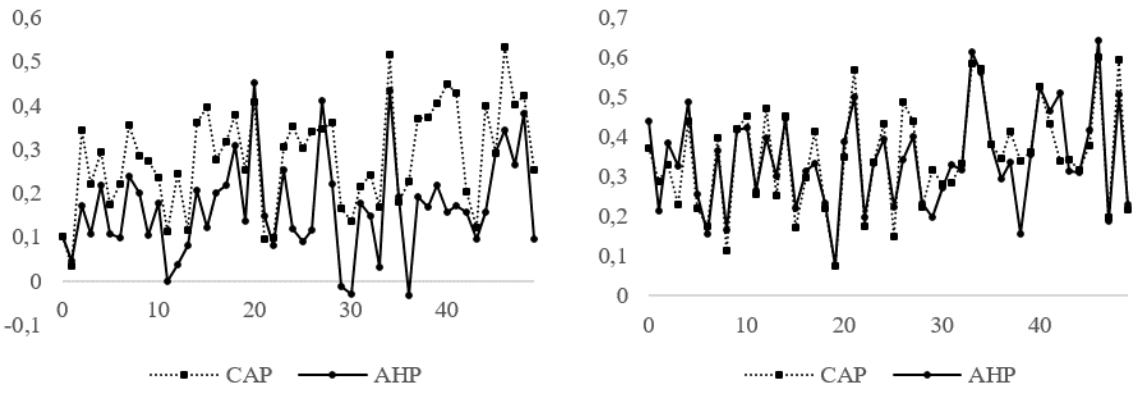

(a) Where the chosen alternative (output) is different (b) Where the chosen alternative (output) is the same

Fig. 2: Comparison between CAP and AHP towards the satisfaction obtained in simulations

Fig $2 \mathrm{~b}$ (X-axis represents the simulation number and Y-axis represents the satisfaction level) shows the results obtained in a sample of 50 simulations of 6767 where the alternative selected by CAP was the same as AHP. These results confirmed that the satisfaction obtained by agents using CAP and AHP was always very similar. Besides this it was verified that the average satisfaction level obtained by CAP in these 6767 simulations was of 0,3484 and AHP was of 0,3429. CAP obtained a higher satisfaction in 3632 simulations while AHP obtained a higher satisfaction in 3135 simulations.

Table 9 shows the results collected for CAP and UF. In 10000 simulations CAP obtained a higher satisfaction in 5448 occasions. The average, standard deviation, maximum, minimum and median values concerned CAP when the satisfaction obtained was higher and corresponded to the difference of satisfaction between CAP and UF (CAP - UF). Likewise, when UF obtained a higher satisfaction those values corresponded to the difference of 
satisfaction between UF and CAP (UF - CAP). It was verified that the average obtained in CAP was almost the same as the average obtained by UF. The standard deviation, minimum, maximum and median values obtained by both methods were also very similar.

Table 9: Comparison between CAP and UF in 10000 simulations

\begin{tabular}{lrr}
\hline & CAP & UF \\
\hline Count & 5448 & 4552 \\
Average & 0,0526 & 0,0451 \\
Standard Deviation & 0,0438 & 0,0358 \\
Maximum & 0,3826 & 0,2656 \\
Minimum & $2,3876 \times 10^{-6}$ & $4,5927 \times 10^{-6}$ \\
Median & 0,0423 & 0,0377 \\
\hline
\end{tabular}

CAP selected a different alternative from UF in 1072 occasions. Fig 3a (X-axis represents the simulation number and Y-axis represents the satisfaction level) shows the results from a sample of 50 simulations. Identical to CAP/AHP comparison, in this situation CAP also obtained a higher satisfaction in most of the times compared to UF. The average satisfaction level obtained by agents in those 1072 simulations was of 0,2840 and UF was only of 0,2365 . It was verified that the average difference was inferior in this situation compared to CAP/AHP comparison. Besides this, CAP obtained a higher satisfaction in 770 out of the 1072 simulations.
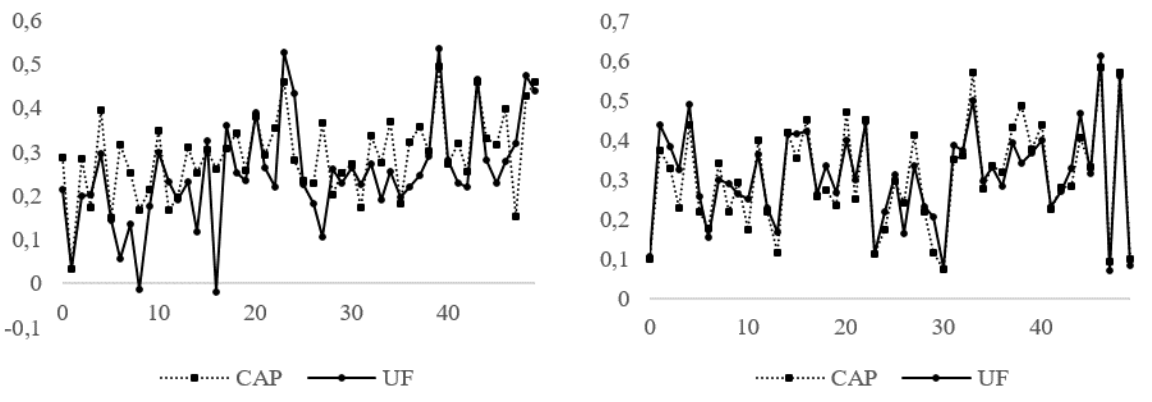

(a) Where the chosen alternative (output) is different (b) Where the chosen alternative (output) is the same

Fig. 3: Comparison between CAP and UF towards the satisfaction obtained in simulations

Fig $3 \mathrm{~b}$ (X-axis represents the simulation number and Y-axis represents the satisfaction level) shows the results obtained in a sample of 50 simulations of 8928 where the alternative selected by CAP was the same as UF. These results were identical to the results obtained in CAP/AHP comparison with the satisfaction obtained by CAP and UF also being very similar. Besides this it was verified that the average satisfaction level obtained by CAP in these 8928 simulations was of 0,3366 and UF was of 0,3332 . CAP obtained a higher satisfaction in 4678 simulations while UF obtained a higher satisfaction in 4250 simulations.

Table 10 shows the results collected for CAP and TOPSIS. In 10000 simulations CAP obtained a higher satisfaction in 5536 occasions. The average, standard deviation, maximum, 
minimum and median values concerned CAP when the satisfaction obtained was higher and corresponded to the difference of satisfaction between CAP and TOPSIS (CAP - TOPSIS). Likewise, when TOPSIS obtained a higher satisfaction those values corresponded to the difference of satisfaction between TOPSIS and CAP (TOPSIS - CAP). It was verified that the average obtained in CAP was almost the same as the average obtained by TOPSIS. The standard deviation, minimum, maximum and median values obtained by both methods were also very similar.

Table 10: Comparison between CAP and TOPSIS in 10000 simulations

\begin{tabular}{lrr}
\hline & CAP & TOPSIS \\
\hline Count & 5536 & 4464 \\
Average & 0,0580 & 0,0460 \\
Standard Deviation & 0,0506 & 0,0366 \\
Maximum & 0,3826 & 0,2589 \\
Minimum & $2,3872 \times 10^{-6}$ & $4,5927 \times 10^{-6}$ \\
Median & 0,0423 & 0,0377 \\
\hline
\end{tabular}

CAP selected a different alternative from TOPSIS in 1506 occasions. Fig 4a (X-axis represents the simulation number and Y-axis represents the satisfaction level) shows the results from a sample of 50 simulations. Identical to CAP/AHP and CAP/UF comparisons, in this situation CAP also obtained a higher satisfaction in most of the times compared to TOPSIS. The average satisfaction level obtained by agents in those 1506 simulations was of 0,2681 and TOPSIS was only of 0,21. It was verified that the average difference was inferior in this situation compared to CAP/AHP comparison and was very similar to the CAP/UF comparison. Besides this, CAP obtained a higher satisfaction in 1063 out of the 1506 simulations.
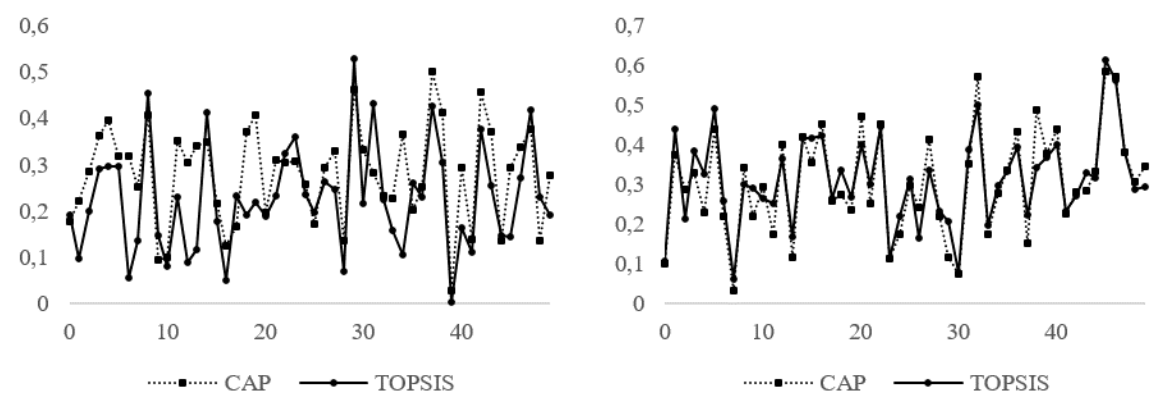

(a) Where the chosen alternative (output) is different (b) Where the chosen alternative (output) is the same

Fig. 4: Comparison between CAP and TOPSIS towards the satisfaction obtained in simulations

Fig $4 \mathrm{~b}$ (X-axis represents the simulation number and Y-axis represents the satisfaction level) shows the results obtained in a sample of 50 simulations of 8494 where the alternative selected by CAP was the same as TOPSIS. These results were identical to the results 
obtained in CAP/UF comparison with the satisfaction obtained by CAP and TOPSIS also being very similar. Besides this it was verified that the average satisfaction level obtained by CAP in these 8494 simulations was of 0,3421 and TOPSIS was of 0,3380 . CAP obtained a higher satisfaction in 4473 simulations while TOPSIS obtained a higher satisfaction in 4021 simulations.

To close the study on this simulation environment (10000 simulations) we compared the number of times when each method obtained the best satisfaction compared with the other methods. Table 11 shows the data collected. It was verified that the satisfaction level obtained was higher by CAP in 5195 simulations, by AHP in 191 simulations, UF in 308 simulations and TOPSIS in only 48 simulations. In situations when more than one method obtained the same highest satisfaction level it was possible to observe that CAP obtained the same result and the remaining methods increased significantly. It was possible to conclude that in 4258 simulations more than one method achieved the same highest satisfaction level. Because of that it was important to figure if CAP obtained much worse results. Average satisfaction values obtained in the 4805 simulations when CAP did not achieve the best results were of $0,2975,0,2833,0,3238$ and 0,3204 for AHP, CAP UF and TOPSIS respectively.

Table 11: Satisfaction comparison between all methods

\begin{tabular}{lcccc}
\hline & AHP & CAP & UF & TOPSIS \\
\hline Best & 191 & 5195 & 308 & 48 \\
Best or Equal & 3516 & 5195 & 4317 & 4272 \\
\hline
\end{tabular}

Another point that was studied was related with the differences between criteria weighting and rankings comparison done by each method. In Table 12, it is presented the values obtained by each method for criteria weighting and rankings comparison in 4 simulations. In each simulation a random agent was selected and used as reference. For this problem (not too complex, with just 4 criteria) it was verified that AHP and TOPSIS obtained the same criteria ranking (in all simulations) even with different criteria weighting. In case of CAP, the criteria ranking was similar to AHP and TOPSIS but with some differences. For example, in the second simulation, CAP classified "Safety" and "Convenience" as VIC while AHP and TOPSIS classified those same criteria differently even though their weighting was very similar. Another interesting fact that was verified was related with how AHP and TOPSIS criteria ranking was always done based on the weighting value which resulted in a sequential order $(1,2,3, \ldots, n)$. In case of CAP criteria ranking this order was not necessarily sequential and there were situations where two or more criteria were given the same rank. For example in the second simulation, CAP ranking classified two criteria as VIC and two criteria as INC in a scale with the following decreasing order: VIC, IC, MC, NIC and INC.

For the last study, a simulation environment was created to understand how the results obtained by each method varied depending on the number of agents (decision-makers) involved in the decision-making process. 1000 simulations were run for each group of decision-makers $(2,4,8,10,20,30,40,50,100,200,300,400$ and 500 agents) totaling 13000 simulations. Similarly to previous procedure, CAP was compared directly with each other method. The main goals were to measure the variation of the average satisfaction level obtained by each method with the variation of the number of decision-makers involved in the process and also measure how that variation brought each method closer according to the number of equivalent decisions. 
Table 12: Example of criteria ranking according to each method

\begin{tabular}{llrrrrrr}
\hline \multirow{2}{*}{ Simulations } & \multirow{2}{*}{ Criteria } & \multicolumn{2}{c}{ CAP } & \multicolumn{2}{c}{ AHP } & \multicolumn{2}{c}{ TOPSIS } \\
& & Weight(\%) & Rank & Weight(\%) & Rank & Weight(\%) & Rank \\
\hline \multirow{3}{*}{1} & Efficacy & - & MC & 14,35 & 3 & 23,15 & 3 \\
& Safety & - & VIC & 58,49 & 1 & 45,37 & 1 \\
& Convenience & - & INC & 4,55 & 4 & 1,85 & 4 \\
& Low cost & - & IC & 22,61 & 2 & 29,63 & 2 \\
\hline \multirow{4}{*}{2} & Efficacy & - & INC & 5,10 & 3 & 0,50 & 3 \\
& Safety & - & VIC & 45,86 & 1 & 49,74 & 1 \\
& Convenience & - & VIC & 43,93 & 2 & 49,24 & 2 \\
& Low cost & - & INC & 5,10 & 3 & 0,50 & 3 \\
\hline & Efficacy & - & VIC & 44,49 & 1 & 43,47 & 1 \\
& Safety & - & INC & 5,16 & 4 & 3,86 & 4 \\
& Convenience & - & NIC & 7,76 & 3 & 9,66 & 3 \\
& Low cost & - & VIC & 42,56 & 2 & 42,99 & 2 \\
\hline \multirow{3}{*}{4} & Efficacy & - & MC & 16,38 & 3 & 25,47 & 3 \\
& Safety & - & VIC & 55,66 & 1 & 43,39 & 1 \\
& Convenience & - & INC & 4,58 & 4 & 0,94 & 4 \\
& Low cost & - & IC & 23,36 & 2 & 30,18 & 2 \\
\hline
\end{tabular}

Fig 5a (X-axis represents the number of agents and Y-axis represents the satisfaction level) shows the average satisfaction level obtained by CAP and AHP (when the achieved decisions were different) for each set of 1000 simulations done with each group of decisionmakers $(2,4,8,10, \ldots, 500)$. It was verified that the satisfaction obtained by AHP was relatively the same throughout each scenario. In case of CAP the average satisfaction level obtained was higher when there were a small number of decision-makers involved in the process and decreased and stayed relatively constant as the number of decision-makers involved in the process increased. However, the average satisfaction level obtained by CAP was always higher compared to AHP in all scenarios, more particularly when the number of decision-makers was less than 20.

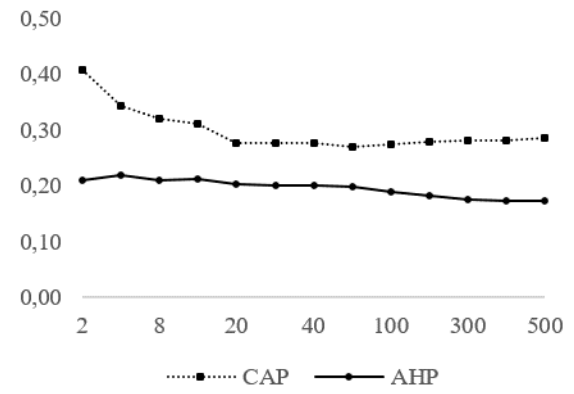

(a) Satisfaction obtained

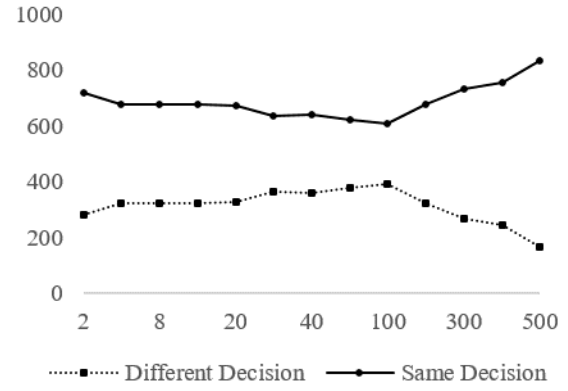

(b) Average number of different and same decision

Fig. 5: Comparison between CAP and AHP

Fig $5 \mathrm{~b}$ (X-axis represents the number of agents and Y-axis represents the number of simulations) shows the number of times in each scenario when CAP and AHP reached same 
or different decisions. It was possible to notice that there were more different decisions when the number of decision-makers involved in the process ranged from 30 to 100. Decisions were least different when 500 decision-makers participated in the process $(83,3 \%)$.

Fig 6a (X-axis represents the number of agents and Y-axis represents the satisfaction level) shows the average satisfaction level obtained by CAP and UF (when the achieved decisions were different) for each set of 1000 simulations done with each group of decisionmakers $(2,4,8,10, \ldots, 500)$. It was verified that the average satisfaction level obtained in both methods was very similar. However, CAP still obtained better results compared with UF in each scenario. In scenarios where more than 100 decision-makers were considered $(200,300,400$ and 500) there was no average satisfaction level obtained as both methods always reached the same decision.

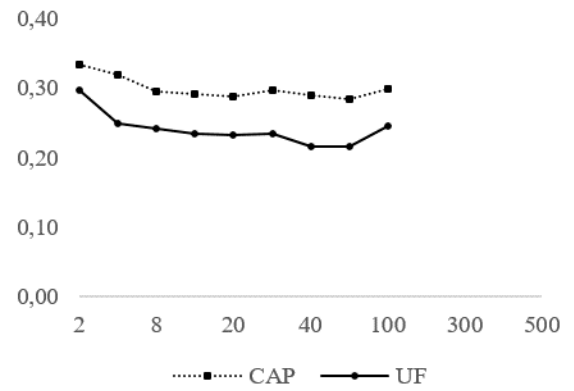

(a) Satisfaction obtained

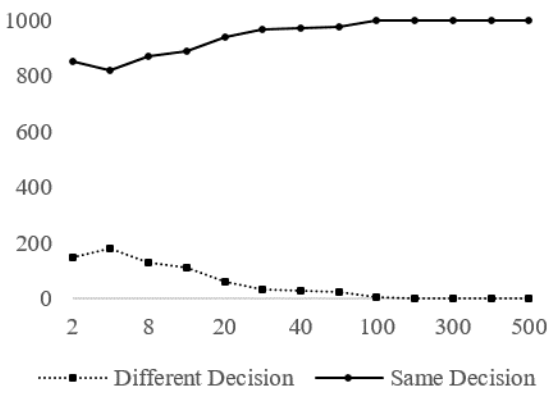

(b) Average number of different and same decisions

Fig. 6: Comparison between CAP and UF

As can be seen in Fig $6 \mathrm{~b}$ (X-axis represents the number of agents and Y-axis represents the number of simulations) when the number of decision-makers involved in the process is higher than 100 both methods always reached the same decision. It was also verified that the most significant difference in the decisions reached by both methods occurred between 2 and 20 participant agents.

Fig 7a (X-axis represents the number of agents and Y-axis represents the satisfaction level) shows the average satisfaction level obtained by CAP and UF (when the achieved decisions were different) for each set of 1000 simulations done with each group of decisionmakers $(2,4,8,10, \ldots, 500)$. Unlike previous methods comparisons, in this situation CAP obtained a lower average satisfaction level than TOPSIS in 2 scenarios (100 and 200 decisionmakers), but still not too relevant. When we considered between 2 and 10 decision-makers participating in the process, CAP obtained an average satisfaction level considerably higher than TOPSIS. When the number of decision-makers was greater than 10, both methods obtained very similar satisfaction levels. In this comparison it was also verified that when there were more than 200 decision-makers participating in the process, both methods reached the same decision.

As can be seen in Fig 7b (X-axis represents the number of agents and Y-axis represents the number of simulations) when the number of decision-makers participating in the process is greater than 200 , both methods always reached the same decision. In the scenario with 200 decision-makers both methods achieved different decisions in just 2 simulations (out of 


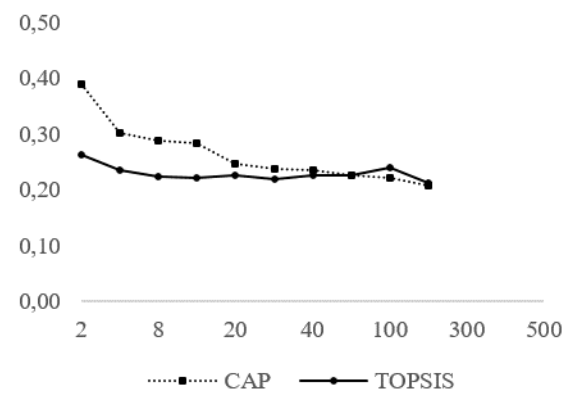

(a) Satisfaction obtained

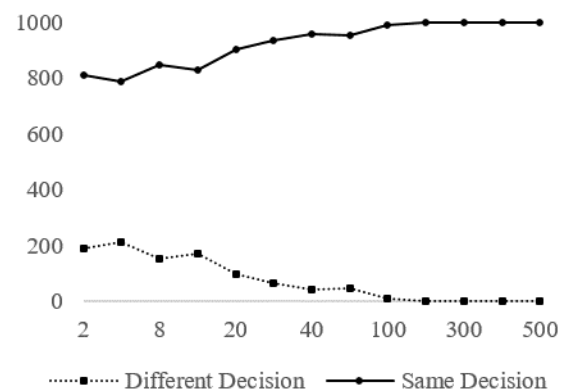

(b) Average number of different and same decisions

Fig. 7: Comparison between CAP and TOPSIS

1000). It was also verified that the most significant difference in the decisions reached by both methods occurred between 2 and 10 participant agents.

\section{Discussion}

Including cognitive aspects in Multiple Criteria Decision Analysis (MCDA) methods is something still inexistent in literature (to the best of our knowledge). Some "not rational" questions are known to affect the decision-making process (Simon, 1987b). The reason why MCDA methods do not consider cognitive aspects might be related to something more important than just not agreeing or accepting their advantage. Which cognitive aspects should be considered? How should they be included in the decision-making process? Etc.

Let us imagine a simple scenario where a family is trying to decide a restaurant to celebrate the anniversary of one of its family members. In this scenario, it is easy to understand the advantage of configuring/modelling not only the preferences towards criteria and alternatives but also the intentions of each family member. It is very likely that the main goal (or main intention) of each member is to please the one whose birthday is being celebrated. Therefore, if the decision reached was choosing a restaurant which is the favourite of some of the family members but on the other hand makes the person whose birthday is being celebrated very unsatisfied, that dissatisfaction will affect all family members and make them think it was a bad decision. Any existing MCDA method is yet not capable to deal with this kind of problem, unlike Cognitive Analytic Process (CAP) which is proposed for the first time in this work.

It was verified that CAP, on average, managed to be almost always superior to any other tested method. CAP had an even more considerable advantage compared with other methods when the decision reached was different from the others presented by those methods. It is important (and somehow interesting) to refer that in situations where the decision reached by all methods was the same, even if CAP considered cognitive aspects, the average satisfaction values obtained were be very similar.

Including the "irrational" component to the typical MCDA rationality provided better results in almost all scenarios that were considered. It was verified that CAP lost many of its benefits when considering a group with more than 20/30 decision-makers. In those situations CAP revealed a very similar performance compared to TOPSIS method or an UF. However, 
this situation was a good example to consider cognitive aspects in CAP correctly. The intentions of each decision-maker in a very large group would be diluted. It is unthinkable to consider using cognitive aspects, for example, for each voter in presidential elections of a country. CAP was advantageous especially when the group decision-makers varied between 2 and 20 elements. This allowed us to conclude that including cognitive aspects could be very irrelevant if we were dealing with very large groups of decision-makers.

It must also not be forgotten that CAP was very easy to configure and did not require more data than what was presented in (Carneiro et al, 2015c). On the other hand, using AHP which is considered to have a very high configuration cost (Dehe and Bamford, 2015) might result in decision-makers refusing or rejecting to use software with such kind of approach.

With this work a new hypothesis of research is raised: The advantages identified in this work by using CAP method will be even more evident in real scenarios. This happens because it is mathematically impossible to define agents that can create and imagine typical intentions of a human being in very specific real contexts.

\section{Conclusion and Future Work}

Nowadays the decisions made by managers and executives are mostly performed in groups. Thereby, group decision-making is a process in which a group of people, called participants, act collectively analyzing a set of variables, considering and evaluating the available alternatives in order to select one or more solutions. The number of participants involved in the process is variable and all of them may be at the same space/place at the same time or either geographically dispersed at different times.

Usually, Multiple Criteria Decision Analysis (MCDA) methods are techniques used to support the decision-making process. MCDA methods allow decision-makers to make a certain multi-criteria problem configuration and based on that configuration a solution will be suggested (supposedly the best). MCDA methods, however, have certain problems (according to literature) due to the completely analytical and rational way in which the multi-criteria problems are considered.

In this work, we presented Cognitive Analytic Process (CAP) which is MCDA method that included cognitive aspects in its analysis and that let decision-makers configure not only their preferences (criteria and alternatives) but also their intentions. CAP allowed decisionmakers to configure a multi-criteria problem (criteria and alternatives) as well as their behaviour style (which could be seen as their position before the problem and the decisionmaking group), their expertise level and select which other decision-maker was credible or not. It also had the advantage of only requiring information that allowed fast configurations (resulting in a very high level of usability) with the particularity that CAP worked even if the decision-maker did not configure any of the points mentioned before. This way, CAP could process all the information and suggested a solution combining the rational component with the irrational dimension (which is sometimes difficult to explain by decision-makers themselves).

The results achieved in this work confirmed the advantage of what was being expressed in literature for many decades. Considering cognitive aspects brought advantages to the group decision-making process and allowed reaching higher quality decisions and with more satisfaction. Moreover, we made this idea computationally feasible. CAP demonstrated that it could almost always be superior than some of the most acknowledged MCDA methods existing in literature. Besides this, CAP demonstrated that it was especially useful in process that included a maximum number of 20 decision-makers. 
As future work, there are still some points to be studied. In first place, we intend to compare CAP with most recent MCDA methods. After that, we intend to compare CAP with those methods in a case of study with real decision-makers, since we believe that in a real environment CAP is capable to be even better than other MCDA methods. Finally, we want to learn with all these processes and work in shaping CAP to make it an even stronger and more complete MCDA method.

Acknowledgements This work was supported by COMPETE Programme (operational programme for competitiveness) within project POCI-01-0145-FEDER-007043, by National Funds through the FCT - Fundação para a Ciência e a Tecnologia (Portuguese Foundation for Science and Technology) within the Projects UID/CEC/00319/2013, UID/EEA/00760/2013, and the João Carneiro PhD grant with the reference SFRH/BD/89697/2012.

\section{References}

Ahn B, Choi S (2012) Aggregation of ordinal data using ordered weighted averaging operator weights. Annals of Operations Research 201(1):1-16

Bates J, et al (1994) The role of emotion in believable agents. Communications of the ACM 37(7):122-125

Bozóki S, Dezső L, Poesz A, Temesi J (2013) Analysis of pairwise comparison matrices: an empirical research. Annals of Operations Research 211(1):511-528

Carneiro J, Marreiros G, Novais P (2015a) Using satisfaction analysis to predict decision quality. International Journal of Artificial Intelligence ${ }^{\mathrm{TM}} 13(1): 45-57$

Carneiro J, Martinho D, Marreiros G, Novais P (2015b) Defining Agents' Behaviour for Negotiation Contexts, Springer, pp 3-14

Carneiro J, Martinho D, Marreiros G, Novais P (2015c) A general template to configure multi-criteria problems in ubiquitous gdss. International Journal of Software Engineering and Its Applications 9:193-206, DOI 10.14257/astl.205.97.17

Carneiro J, Santos R, Marreiros G, Novais P (2015d) Ubigdss: A theoretical model to predict decision-makers' sat-isfaction. International Journal of Multimedia and Ubiquitous Engineering 10(7):191-200

Castelfranchi C (1994) Guarantees for autonomy in cognitive agent architecture. In: Intelligent agents, Springer, pp 56-70

Chen CT (2000) Extensions of the topsis for group decision-making under fuzzy environment. Fuzzy sets and systems 114(1):1-9

De VT (1993) Presenting clinical pharmacology and therapeutics: a problem based approach for choosing and prescribing drugs. British journal of clinical pharmacology 35(6):581586

Dehe B, Bamford D (2015) Development, test and comparison of two multiple criteria decision analysis (mcda) models: A case of healthcare infrastructure location. Expert Systems with Applications 42(19):6717-6727

Dennis AR (1996) Information exchange and use in small group decision making. Small Group Research 27(4):532-550

Edwards W (1954) The theory of decision making. Psychological bulletin 51(4):380

Falcone R, Castelfranchi C (2001) Social trust: A cognitive approach. In: Trust and deception in virtual societies, Springer, pp 55-90

Fenton-O'Creevy M, Soane E, Nicholson N, Willman P (2011) Thinking, feeling and deciding: The influence of emotions on the decision making and performance of traders. Journal of Organizational Behavior 32(8):1044-1061 
Figueira J, Greco S, Ehrgott M (2005) Multiple criteria decision analysis: state of the art surveys, vol 78. Springer Science \& Business Media

Flanagin AJ, Metzger MJ (2008) Digital media and youth: Unparalleled opportunity and unprecedented responsibility. Digital media, youth, and credibility pp 5-27

Frith CD, Singer T (2008) The role of social cognition in decision making. Philosophical Transactions of the Royal Society of London B: Biological Sciences 363(1511):38753886

Golmohammadi D, Mellat-Parast M (2012) Developing a grey-based decision-making model for supplier selection. International Journal of Production Economics 137(2):191200

Huber GP (1984) Issues in the design of group decision support sytems. MIS quarterly pp 195-204

Kiesler S, Siegel J, McGuire TW (1984) Social psychological aspects of computer-mediated communication. American psychologist 39(10):1123

Kou G, Wu W (2014) Multi-criteria decision analysis for emergency medical service assessment. Annals of Operations Research 223(1):239-254

Levy G (2007) Decision making in committees: Transparency, reputation, and voting rules. The American Economic Review pp 150-168

Luthans F (2011) Organizational behavior. McGraw-Hill/Irwin 46:594, DOI 10.1146/ annurev.psych.46.1.59

Martinho D, Carneiro J, Marreiros G, Novais P (2015) Dealing with agents' behaviour in the decision-making process. In: Workshop Proceedings of the 11th International Conference on Intelligent Environments, IOS Press, vol 19, p 4

Ogiela L, Ogiela MR (2014a) Cognitive systems and bio-inspired computing in homeland security. Journal of Network and Computer Applications 38:34-42

Ogiela L, Ogiela MR (2014b) Cognitive systems for intelligent business information management in cognitive economy. International Journal of Information Management 34(6):751-760

Rahim MA, Magner NR (1995) Confirmatory factor analysis of the styles of handling interpersonal conflict: first-order factor model and its invariance across groups. Journal of applied psychology 80(1):122

Ram C, Montibeller G, Morton A (2011) Extending the use of scenario planning and mcda for the evaluation of strategic options. Journal of the Operational Research Society 62(5):817-829

Saaty TL (1988) What is the analytic hierarchy process? Springer

Saaty TL (2008) Decision making with the analytic hierarchy process. International journal of services sciences 1(1):83-98

Schwarz N (2000) Emotion, cognition, and decision making. Cognition \& Emotion 14(4):433-440

Schwenk CR (1988) The cognitive perspective on strategic decision making. Journal of management studies 25(1):41-55

Simon HA (1959) Theories of decision-making in economics and behavioral science. The American economic review 49(3):253-283

Simon HA (1965) Administrative behavior, vol 4. Cambridge Univ Press

Simon HA (1979) Rational decision making in business organizations. The American Economic Review 64:493-514, DOI 10.2307/1808698

Simon HA (1986) Rationality in psychology and economics. Journal of Business pp S209S224 
Simon HA (1987a) Making management decisions: The role of intuition and emotion. The Academy of Management Executive (1987-1989) pp 57-64

Simon HA (1987b) Making management decisions: The role of intuition and emotion. The Academy of Management Executive (1987-1989) pp 57-64

Smith ER, Conrey FR (2007) Agent-based modeling: A new approach for theory building in social psychology. Personality and social psychology review 11(1):87-104

Tavana M, Sodenkamp MA, Suhl L (2010) A soft multi-criteria decision analysis model with application to the european union enlargement. Annals of Operations Research 181(1):393-421

Tzeng GH, Huang JJ (2011) Multiple attribute decision making: methods and applications. CRC press

Wang J (2012) Robust optimization analysis for multiple attribute decision making problems with imprecise information. Annals of Operations Research 197(1):109-122 